\title{
THEORY, EXPERIMENTAL REALIZATION, AND PROPERTIES OF MINIATURE BRAGG FIBERS
}

\author{
J.G.Fleming, Shawn-Yu Lin, and Ronald Hadley \\ MEMS and Novel Silicon Technologies, Sandia National Laboratories \\ Albuquerque, NM, 87185-1080
}

\begin{abstract}
This paper outlines our work on the theory, experimental realization and properties of miniature Bragg fibers. These structures were proposed over 20 years ago and consist of hollow tubes in which light is guided in a low index media (air) and confined by surrounding Bragg mirror stacks. The thicknesses of high and low index layers making up the mirror stacks have been determined theoretically. Structures with internal diameters from 5 to 30 microns have been fabricated and much larger structures should also be possible. We have demonstrated the fabrication of these structures with short wavelength band edges ranging from 400 to $1600 \mathrm{~nm}$. There may be potential applications for such structures in the fields of integrated optics and BioMEMS.
\end{abstract}

\section{INTRODUCTION}

The propagation of long wavelength, millimeter scale radiation through hollow waveguides is well known. Less well know are proposals in the 1960's to used hollow metal structures to propagate short wavelength light [1]. This field died out with the development of glass optical fibers. There is also interest in various coated hollow metal fibers for the propagation of 10micron radiation for medical applications [2-6]. The inside hollow diameter of these metallic structures is typically on the order of a millimeter. Yariv and coworkers first proposed Bragg fibers in 1978 [7]. Several other groups have recently revisited the idea of Bragg fibers [8-10]. In most cases the application being considered is that of optical fibers. Such fibers potentially have very low losses, in theory considerably less than that of standard silica fibers. However, this extremely low loss behavior is only exhibited by a particular mode, the $\mathrm{TE}_{01}$, which does not propagate in standard fibers. In our work we are interested in the development of miniature Bragg fibers with inner diameters in the order of 1200 microns. These fibers are fabricated in the plane of the wafer using modifications of standard silicon processes. We are interested not in the very low loss applications associated with optical fiber communications, but rather applications associated with integrated optics and BioMEMS where losses on the order of $1-5 \mathrm{~dB} / \mathrm{cm}$ may be acceptable. Potential advantages of this approach in the field of integrated optics include: elimination of end facets, ease of integration with index matching and other fluids and possible development of novel thermo-optical structures. Potential advantages in the area of BioMEMS include the guiding of light and liquids in the same platform.

\section{THEORY}

Typical waveguides guide light as the result of a difference in refractive index between two low loss optical media. Light is confined in such structures to the higher index media. The greater the index contrast between the low and high index media the greater the level of confinement. The greater the confinement the smaller the radius of curvature which can be defined without loss of light from the guide. The smaller the radius of curvature the smaller the size of the final part. However when light is tightly confined, its mode in the waveguide is very small and it becomes difficult to couple light into and out of the low index contrast optical fibers, which are the backbone of optical communications. The guiding principle of Bragg fibers is fundamentally different. In this case light propagates in a low index material, air, and is confined by a dielectric mirror. Compact bends can be realized using dielectric mirrors. The mode size for single mode propagation is on the order of a micron. However, in certain cases these structures can be made effectively single mode [9]. The efficiency of the mirror stacks used to confine the light is dependent on the index contrast between the high and low index materials making up the mirror. In the case of high index contrast systems such as silicon $(\mathrm{n} \sim 3.5)$ and silicon dioxide $(\mathrm{n} \sim 1.46)$ only three pairs of layers result in reflectivity's greater than those of metals over a very large range of wavelengths. Also, in the case of these high index contrast systems, the mirrors are effective for all angles of incidence. The thickness of higher refractive index layers making up the mirror stack is very similar to those given by that of a 1/4-wave stack, the wavelength divided by four times the index of refraction. Optimal layer thickness targeted at a wavelength of 1.5 microns lower wavelength band edge, are 0.36 microns for $\mathrm{SiO}_{2}, 0.11$ microns for $\mathrm{Si}$ and 0.21 microns for $\mathrm{SiN}$. Such an arrangement results in low losses in the TE mode, but high losses for TM modes. TM mode losses can be reduced without greatly increasing TE mode losses by doubling the thickness of the inner most layer. Losses for these dimensions at 1.5 micron wavelengths for a 4 pair $\mathrm{Si} / \mathrm{SiO} 2$ mirror stack are predicted to be on the order of $3 \mathrm{~dB} / \mathrm{cm}$ for TM and $0.2 \mathrm{~dB} / \mathrm{cm}$ for TE. Losses can be reduced by increasing the number of layers and can be greatly reduced by propagating only the $\mathrm{TE}_{01}$ mode in a $\sim 13$ micron diameter circular Bragg fiber [9].

\section{EXPERIMENTAL DETAILS}

The structures were fabricated on 6-inch silicon wafers using widely available silicon processing tools. The process steps can generally be classified as the creation of an undercut trench, the sealing of the top of the trench, and the deposition of the mirror stack, Figure 1. The materials making up the mirror stack are introduced into the trench through periodic openings along it's length. The openings are widely enough spaced that they do not seem to effect the propagation of light along the trench to first order. The process relies on low sticking constant (high step coverage) of CVD (chemical vapor deposition) and diffusion processes, in particular the deposition of $\mathrm{Si}$ from silane, $\mathrm{SiN}$ from dichlorosilane and ammonia and the thermal oxidation of silicon. Combinations of processes enable the creation of $\mathrm{Si}, \mathrm{SiN}$ and $\mathrm{SiO}_{2}$ mirror layers. In Bragg fibers, the range of wavelengths propagated depends on the layer thickness. Since the thickness is determined by a CVD process we have very tight control over these parameters and it is readily possible to create waveguides which propagate short wavelength radiation. It is also noteworthy that no fine 
feature lithography is required to generate the structures. Relatively simple additions to the process can be employed to modify the tube shape, Figure 2. Since the mirror levels are on the internal surfaces of the channel it is also possible to remove the opaque layers surrounding the structure, Figure 3.

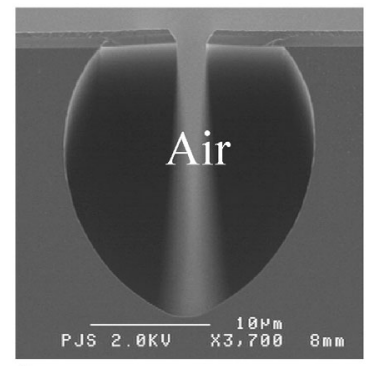

1a

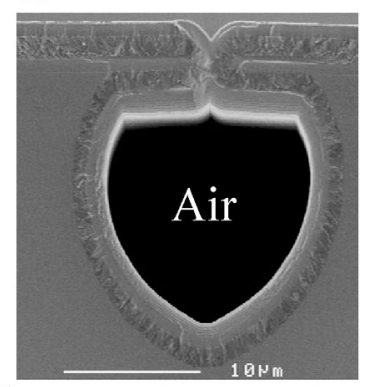

$1 \mathrm{c}$

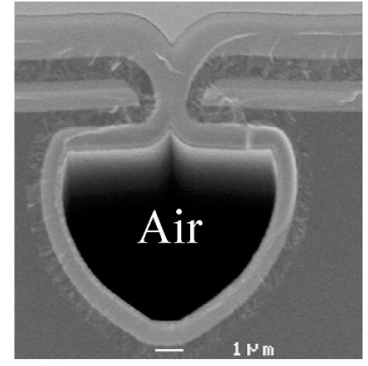

$1 b$

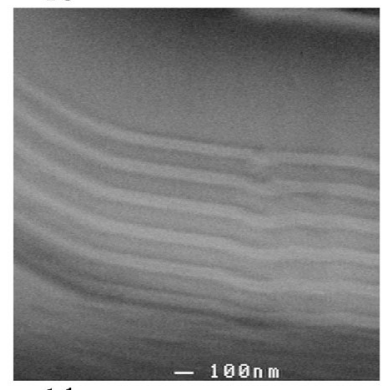

$1 d$
Figure 1. Scanning electron micrograph cross sections outlining the steps in the fabrication of miniature Bragg fibers. la shows the formation of the undercut trench. It is formed by isotropically etching the Si substrate through an open line in a low stress SiN layer. The isotropic etch is $F$ based. Figure $1 b$ shows the filling of the opening in the silicon nitride. This is achieved by first depositing 2 microns of polysilicon and then growing a 1-micron thermal oxide. Figure 1c shows the structure after the deposition of the mirror stack. Figure 1d shows a magnified image of a mirror stack consisting of $\mathrm{SiO}_{2}$ and $\mathrm{SiN}$ targeted at 400nm. The reactants needed for the deposition of the mirror stacks enter the trenches through periodic, larger openings not shown in this view, which are not closed off by the polysilicon and oxidation processes of step $b$.
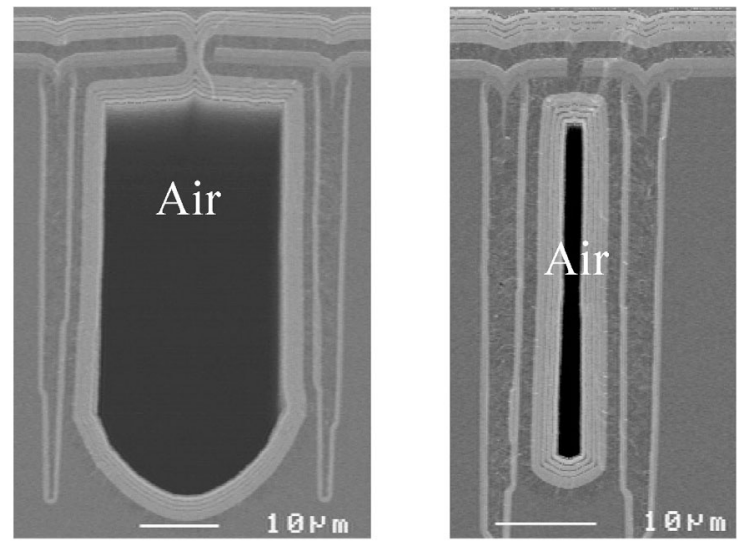

Figure 2. By adding a second mask level which is used to form oxide-lined trenches it is possible to control the lateral dimension of the trench. By controlling the width of the trench opening and the etch time it is possible to control the depth of the trench.

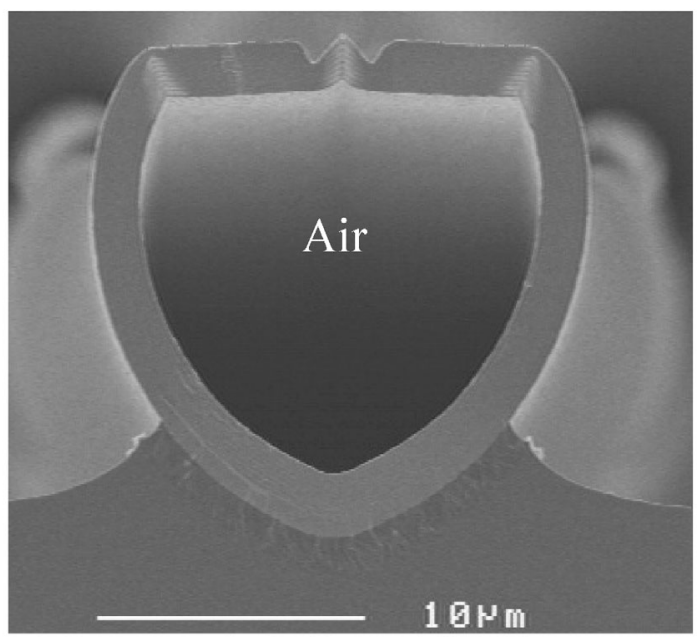

Figure 3. Since the mirror stack is located on the internal surfaces of the tube it is possible to blanket etch back the top layers to remove the optically opaque polysilicon layer used to close the top seam of the trench and, if desired, silicon from the trench edges. This will enable the propagation of light outside the "bandgap" of the mirror stack through the structure.

\section{TESTING}

The optical properties of the structures were tested by the free space coupling of laser light into them. Tunable laser modules were used to investigate the wavelength dependence of light propagation through the channels. Losses were determined by investigating the variation of light intensity through various lengths of Bragg fiber. In the case of structures designed to propagate visible light, simple experiments were performed in which light from a tungsten white light source was propagated through the channels and the resulting image was captured on film. In all cases, the lengths of channel investigated ranged from 100's of microns to millimeters.

\section{RESULTS AND DISCUSSION}

Figure 4 demonstrates the propagation of visible light through miniature Bragg fibers designed to have a short wavelength cutoff of 400nm. The light source was a white light tungsten bulb and the length of channel was roughly 6 millimeters.

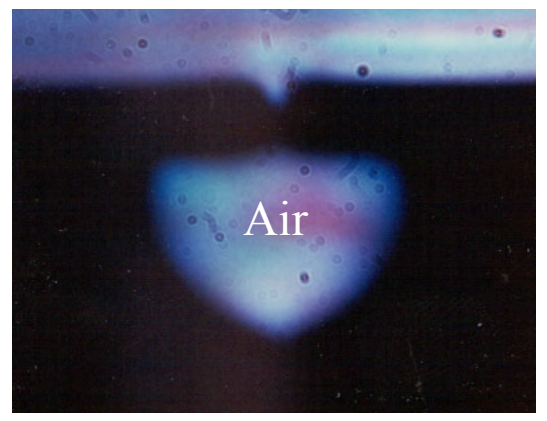

Figure 4. Optical image of visible light propagating through roughly 6 millimeters of a miniature Bragg fiber. The magnification is 1000 times. The Bragg fiber was targeted to have a lower wavelength cutoff at 400nm. The mirror stack consisted of $\mathrm{SiN}$ and $\mathrm{SiO}_{2}$. 
Figure 5 shows a plot of light intensity as a function of wavelength for two different lengths of Bragg fiber with a lower wavelength edge at $\sim 1550 \mathrm{~nm}$. In this case the mirror layers were $\mathrm{Si}$ and $\mathrm{SiN}$ and the diameter was roughly 15 microns and there were 4 mirror pairs.

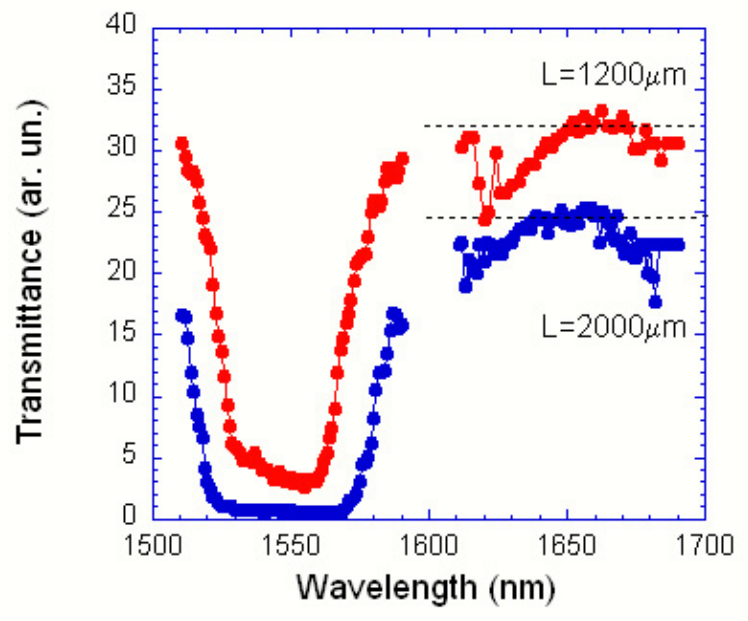

Figure 5. Transmission as a function of wavelength for two different lengths of Bragg fiber. The lower band edge is at $1550 \mathrm{~nm}$. The discontinuity at $\sim 1600 \mathrm{~nm}$ is the result of changing laser modules. The exact long wavelength cutoff is unknown since the series of laser modules available did not extend further than $1680 \mathrm{~nm}$.

From data similar to that presented in Figure 5 we estimate the losses in these structures to be on the order of $2-5 \mathrm{db} / \mathrm{cm}$. This is in good agreement with that predicted by theory. The losses of the shorter wavelength structures of Figure 4 have not yet been measured, however, we believe the losses will be considerably lower. Modeling indicates that much of the loss in $\mathrm{Si} / \mathrm{SiN}$ mirror stacks is the result of adsorption by the polysilicon in the mirror stack. The value of polysilicon adsorption used in the theory was $10 \mathrm{~dB} / \mathrm{cm}$. The adsorption coefficients of silicon dioxide and silicon nitride are much lower.

Since the range of transmission of the structures exceeded the range of the available turnable laser modules we were unable to accurately identify the full bandwidth of the Bragg fibers tested in Figure 5. However, the $100 \mathrm{~nm}$ demonstrated is more than adequate for many applications. It is also notable that the drop off of intensity at $\sim 1550 \mathrm{~nm}$ evident in Figure 5 is very sharp.

Since the light in these structures is propagating in air there is no discontinuity in index as the light from the laser enters and leaves the Bragg fiber. As a result the Fabry-Perot resonant features typically present are, in this case, entirely absent.

We have observed experimentally that the coupling of laser light into the miniature Bragg fibers is relatively insensitive to the alignment of the beam with the centerline of the fiber, making laser coupling to the Bragg fibers relatively straightforward. Theory predicts that, for the Bragg fibers to be single mode, the diameter of the tube should be on the order of a micron. While this should be possible it may be relatively difficult to achieve with the current fabrication process. The roughly 15 micron diameter structures fabricated to date are multimode. It has been theoretically predicted that circular structures with similar dimensions could be made to be effectively single mode. Losses of the $\mathrm{TE}_{01}$ mode are predicted to be significantly less than that of other modes [9]. Thus during propagation over long distances all the other modes will dissipate leaving only the $\mathrm{TE}_{01}$ mode. It should be possible to fabricate quite circular miniature Bragg fiber structures; however, this mode is not typically generated by light emitting devices. While the integrated mode size of the miniature Bragg fibers can be made to match that of a single mode fiber the actual mode shape will be different. This may be an issue for integrated optics applications. We are in the process of experimentally determining what effect this has on the input and output coupling of such structures to fibers and whether or not it is possible to geometrically come up with structures which will only support a single mode.

We have also investigated the formation of both splitters and bends, which will be important building blocks of any real device. Both have been demonstrated. Bends with radii of 40 microns have less than $0.4 \mathrm{~dB}$ loss per 90 -degree bend. Mirror bends taking up roughly the same area have similar performance.

The presence of the gas inlet ports along the structure at intervals ranging from 400 to 1600 microns did not appear to have a major influence on loss. This is not unexpected since the openings are well spaced and the "air gaps" are roughly the same size as the hollow tubes.

\section{POTENTIAL APPLICATIONS}

While these structures are still being evaluated, we believe that they may have many potential applications to integrated optics and BioMEMS. One of the major issues in large optical switching arrays is the divergence of light as it propagates in air. This leads to large mirror sizes and requires collection, focusing, alignment and collimator schemes. In miniature Bragg fibers, light also propagates in air, however the light is confined by the Bragg fiber. It may be possible to integrate an array of crossing Bragg fibers with a series of movable, two-position mirror MEMS devices. The MEMS mirrors could fit into the gas inlet ports. In this $\mathrm{N}^{2}$ structure the light would be confined to the Bragg fiber. The mirrors would therefore only need to be on the order of the diameter of the fiber, 5-10 microns. It may even be possible to fabricate very large, $\sim 125$ micron diameter, Bragg fibers into which the optical fiber can be inserted. The fibers can then be adiabatically tapered down to 5 micron in diameter. This approach would eliminate the need for collimators, lensing and other fiber alignment structures. Components of such a system are currently in development.

At present, we have only demonstrated the propagation of light in "air-filled" Bragg fibers. However, it should also be possible to introduce low index liquids into such structures. Many potentially interesting fluids exist. For example, certain fluids exhibit changes of index with temperature, which are an order of magnitude greater than those of solids [11]. It may be possible to integrate fluid filled Bragg fibers with heaters to make more efficient thermo-optical devices such as switches and attenuators.

Finally, we believe that it may be possible to fill such structures with biological fluids. In these structures both light and liquids would be guided within a single platform. At present there exist conventional structures where light, confined in dielectric waveguides, is evanescently coupled into fluorescently tagged biological samples [12-13]. Light emitted from the tags is then 
evanescently coupled back into the waveguide. Both of these evanescent coupling processes are relatively inefficient. On the other hand if light and fluid could be confined to a single channel the interaction of light with the liquid and the trapping of the emitted light in the channel could be much more efficient.

\section{SUMMARY AND CONCLUSIONS}

Miniature Bragg fibers have been demonstrated for the first time. The structures are fabricated using standard silicon processing tools on 6-inch wafers. A wide range of different geometry of structure has been demonstrated as have a number of different mirror stack configurations. Guiding of light from $~ 400$ to $1700 \mathrm{~nm}$ has been demonstrated as have bends and splitters. Losses are $2-5 \mathrm{~dB} / \mathrm{cm}$ and potentially can be many orders of magnitude lower. There are many potentially interesting applications for these novel structures.

\section{ACKNOWLEDGEMENTS}

The authors gratefully acknowledge the efforts of their coworkers at Sandia National Laboratories Microelectronics Development Laboratory and the Compound Semiconductor Research Laboratory, especially Melanie Tuck and James Bur. Sandia is a multiprogram laboratory operated by Sandia Corporation, a Lockheed Martin Company, for the United States Department of Energy.

\section{REFERENCES}

1. E.A.J. Marcatili and R.A. Schmeltzer, "Hollow Metallic and Dielectric Waveguides for Long Distance Optical Transmission and Lasers", Bell System Tech. J., July (1964), 1783.

2. M. Miyagi and S. Kawakami, "Design Theory of DielectricCoated Circular Metallic Waveguides for Infrared Transmission", J. Lightwave Tech., LT-2, 116 (1984).

3. N. Croitoru, J. Dror and I. Gannot, "Characterization of hollow fibers for the transmission of infrared radiation", Appl. Optics, 29, 1805 (1990).

4. Y. Kato and M. Miyagi, "Modes and Attenuation Constants in Circular Hollow Waveguides with Small Core Diameters for the Infrared", IEEE Trans. Microwave Theory and Tech., 40, 679 (1992).

5. F.E. Vermeulen, T. Wang C.R.James and A.M. Robinson, "Propagation of Infrared Radiation in Hollow Microstructural Cylindrical Waveguides", J. Lightwave Tech., 11, 1956 (1993).

6. R. L. Kozodoy, A.T. Pagkalinawan and J. A. Harrington, "Small-bore hollow waveguides for delivery of 3-vm laser radiation", Appl. Optics, 35, 1077 (1996).

7. P.Yeh, A. Yariv and E. Marom, "Theory of Bragg fiber", J. Opt. Soc. Am. 68, 1196 (1978).

8. Y. Xu, R.K. Lee and A. Yariv, "Asymptotic analysis of Bragg fibers", Optics Lett., 25, 1756 (2000).

9. S.G. Johnson et-al, "Low-loss asymptotically single-mode propagation in large-core OmniGuide fibers", Optics Exp., 9, 748 (2001).
10. T. Kawanishi and M. Izutsu, "Coaxial periodic optical waveguide", Optics Exp., 7, 10 (2000).

11. M.B.J. Diemeer, J.J. Brons and E.S. Trommel, "Polymeric optical waveguide switch using the thermooptic effect", J. Lightwave Tech., 7, 449 (1989).

12. T.R.E. Simpson, D.J. Revell, M.J. Cook and D.A. Russell, Evanescent Wave Exited Fluorescence from Self-Assembled Phthalocyanine Monolayers", Langmuir, 13, 460 (1997).

13. H.P. Kao and J.S. Schoeniger, "Hollow cylindrical waveguides for use as evanescent fluorescence-based sensors: effect of numerical aperture on collected signal", Appl. Optics, 36, 8199 (1997). 